\title{
Der Zahnarzt hat's sofort erkannt
}

- Seit mehr als einem Jahr bemerkt eine Patientin rechts am Kinn eine Hautläsion, aus der sich gelegentlich seröse Flüssigkeit entleerte. Therapieversuche mit Steroidsalben beim Hausarzt waren ohne Erfolg.

Das Aussehen und die fehlende Wachstumstendenz machten eine maligne Läsion unwahrscheinlich. Ein findiger Arzt schickte die Frau zum Zahnarzt, wo sie lange Zeit nicht mehr gewesen sein musste. Jedenfalls sah der Zahnstatus ziemlich desolat aus. Der Zahnarzt stellte die Blickdiagnose einer Zahnfistel, die dann auch operativ beseitigt wurde.

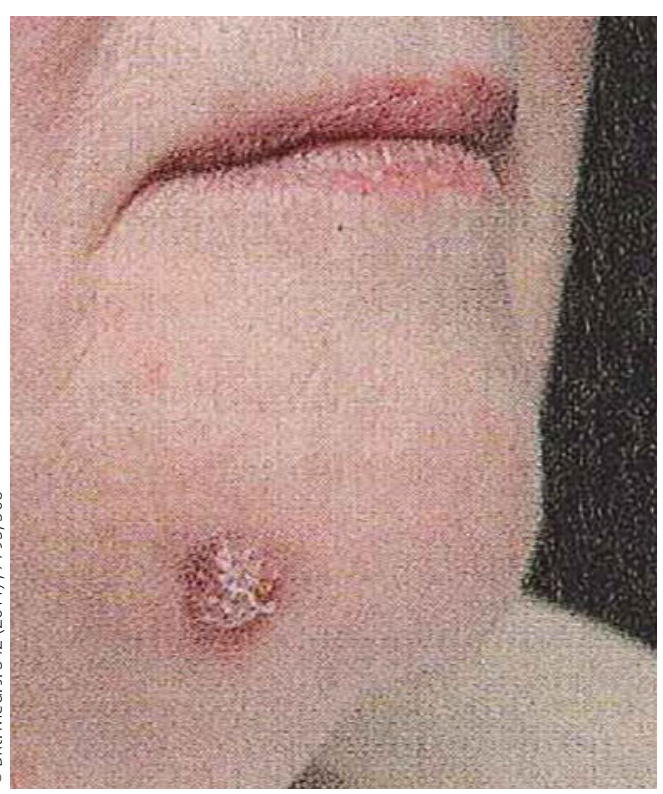

Wieder ein Beleg für die schmerzlich empfundene Tatsache, dass man eben nur an die Dinge denkt, die man auch kennt und die man sozusagen auf dem Radarschirm hat. Jede schlecht heilende Läsion im Bereich des Unterkiefers, die nicht maligne aussieht und sich nicht so verhält, sollte an eine Zahnfistel denken lassen.

H. S. FüESSL =

- S. Sidhu, A.D. Morris

(Susana-sidhu@docotrs.org.uk) The Welsh Institute of Dermatology, University Hospital of Wales, Cardiff CF14 4XW. Brit. Med. J. 342 (2011) , 7795, 506

Der Zahnarzt erkannte die richtige Diagnose auf einen Blick.

\section{Mehr Ösophaguskarzinome unter Bisphosphonaten}

\section{Ob Bisphosphonate das Risiko für ein Ösophagus- bzw. Magenkarzinom erhöhen, wird seit vielen Jahren kontrovers diskutiert. Eine neuere Studie gibt jetzt Aufschluss darüber, ab welcher Therapiedauer mit einem erhöhten Ösophaguskarzinomrisiko gerechnet werden muss.}

— In einer Fallkontrollstudie wurde die Verschreibung von Bisphosphonaten prospektiv erhoben. Anschließend wurden die Daten von 2594 Patienten mit einem Ösophaguskarzinom, 2018 Patienten mit einem Magenkarzinom und 10641 Patienten mit einem kolorektalen Karzinom analysiert. Die Auswertung ergab, dass die Inzidenz des Ösophaguskarzinoms bei Patienten mit einer oder mehreren vorangegangenen Verschrei- bungen von oralen Bisphosphonaten erhöht war. Ein deutlicher Anstieg fand sich bei Patienten mit zehn und mehr Verschreibungen eines Bisphosphonates.

Was den zeitlichen Ablauf betrifft, so stieg die Karzinomrate signifikant erst nach einer dreijährigen Therapie an. Insgesamt war das Ösophaguskarzinomrisiko unabhängig davon, welches Bisphosphonat verordnet wurde. Die Häufigkeit von Magenkarzinomen bzw. kolorektalen Karzinomen war nicht mit der Einnahme von Bisphosphonaten assoziiert.

\section{- J. Green et al.}

Oral bisphosphonates and risk of cancer of esophagus, stomach, and colorectum: Casecontrol analysis within a UK primary care cohort. Brit. Med. J. 341 (2010) c4444

\section{Kommentar}

Dass Bisphosphonate vor allem bei unsachgerechter Einnahme entzündliche Veränderungen im distalen Ösophagus verursachen können, ist hinreichend bekannt. Dass dies das Ösophaguskarzinomrisiko erhöht, dürfte nach diesen Daten kaum noch bezweifelt werden. Doch in absoluten Zahlen gesehen ist die Risikozunahme sehr gering. Während ohne Bisphosphonattherapie ein Ösophaguskarzinom bei 60-79-jährigen Patienten innerhalb von fünf Jahren bei 1 von 1000 auftritt, steigt dieses Risiko bei Einnahme von Bisphosphonaten auf 2 pro 1000.

P. StiefelHAGEN - 